\title{
Synaptotagmin-1 may be a distance regulator acting upstream of SNARE nucleation
}

\author{
Geert van den Bogaart ${ }^{1}$, Shashi Thutupalli ${ }^{2}$, Jelger H Risselada ${ }^{3}$, Karsten Meyenberg ${ }^{4}$, Matthew Holt ${ }^{1}$, \\ Dietmar Riedel $^{5}$, Ulf Diederichsen ${ }^{4}$, Stephan Herminghaus ${ }^{2}$, Helmut Grubmüller ${ }^{3} \&$ Reinhard Jahn ${ }^{1}$
}

\begin{abstract}
Synaptotagmin-1 triggers $\mathrm{Ca}^{2+}$-sensitive, rapid neurotransmitter release by promoting interactions between SNARE proteins on synaptic vesicles and the plasma membrane. How synaptotagmin-1 promotes this interaction is unclear, and the massive increase in membrane fusion efficiency of $\mathrm{Ca}^{2+}$-bound synaptotagmin-1 has not been reproduced in vitro. However, previous experiments have been performed at relatively high salt concentrations, screening potentially important electrostatic interactions. Using functional reconstitution in liposomes, we show here that at low ionic strength SNARE-mediated membrane fusion becomes strictly dependent on both $\mathrm{Ca}^{2+}$ and synaptotagmin-1. Under these conditions, synaptotagmin-1 functions as a distance regulator that tethers the liposomes too far from the plasma membrane for SNARE nucleation in the absence of $\mathrm{Ca}^{2+}$, but while bringing the liposomes close enough for membrane fusion in the presence of $\mathrm{Ca}^{2+}$. These results may explain how the relatively weak electrostatic interactions between synaptotagmin-1 and membranes substantially accelerate fusion.
\end{abstract}

Presynaptic nerve terminals convert electrical signals into chemical signals that target other neurons or somatic cells. This conversion is mediated by depolarization of the plasma membrane, which causes an influx of $\mathrm{Ca}^{2+}$ through voltage-activated $\mathrm{Ca}^{2+}$ channels. The increase in the local cytoplasmic $\mathrm{Ca}^{2+}$ concentration triggers the submillisecond fusion of synaptic vesicles with the plasma membrane. Membrane fusion is mediated by the assembly of SNARE proteins including the R-SNARE synaptobrevin-2 (also known as VAMP2) on the synaptic vesicle and the Q-SNAREs syntaxin-1A and SNAP-25 on the plasma membrane ${ }^{1,2}$. SNARE assembly involves the conserved, membraneadjacent SNARE motifs, proceeds from the $\mathrm{N}$ terminus toward the $\mathrm{C}$-terminal membrane anchors, and results in the formation of a tight coiled-coil structure that pulls the membranes together and overcomes the energy barrier to membrane fusion.

The synaptic vesicle protein synaptotagmin-1 acts as a major $\mathrm{Ca}^{2+}$ sensor for neuronal exocytosis ${ }^{3,4}$. The $65-\mathrm{kDa}$ protein contains a single transmembrane domain followed by a large cytoplasmic domain consisting of a 61-residue unstructured linker and tandem C2-type domains. These $\mathrm{C} 2$ domains, $\mathrm{C} 2 \mathrm{~A}$ and $\mathrm{C} 2 \mathrm{~B}$, bind two and three $\mathrm{Ca}^{2+}$ ions, respectively, with low affinity $(60 \mu \mathrm{M}-1 \mathrm{mM})^{5,6}$ and interact with both anionic membranes and SNARE proteins. Synaptotagmin-1 binds to anionic lipids both in the absence of $\mathrm{Ca}^{2+}$, through a 'polybasic patch' consisting of four lysines on the $\mathrm{C} 2 \mathrm{~B}$ domain ${ }^{7-9}$, and in the presence of $\mathrm{Ca}^{2+}$, through its $\mathrm{Ca}^{2+}$-binding sites ${ }^{5,8-13}$. Anionic lipids such as phosphoserine and phosphatidylinositol 4,5-biphosphate (PtdIns $\left.(4,5) \mathrm{P}_{2}\right)$ complete the $\mathrm{Ca}^{2+}$-binding sites of the $\mathrm{C} 2$ domains and thereby increase the affinity of synaptotagmin- 1 for $\mathrm{Ca}^{2+}$ binding $5,7,8,14$. $\mathrm{Ca}^{2+}$-synaptotagmin-1 locally increases membrane curvature, which suggests that synaptotagmin-1 might act by increasing the membrane tension at the sites of fusion ${ }^{15,16}$. Synaptotagmin-1 also binds directly to SNAP-25, syntaxin-1A and the binary and ternary SNARE complexes ${ }^{3,17-19}$. A model is emerging wherein synaptotagmin-1 structurally changes both the SNARE complex and the membranes in a $\mathrm{Ca}^{2+}$-dependent manner, thereby levering the protein, bending the membrane or both, which induces membrane fusion $^{3,9,11,15-22}$. In these models, synaptotagmin-1 acts downstream of SNARE nucleation, at a fusion-arrested state where the membranes are tethered by a trans SNARE complex in which at least part of the cytoplasmic SNARE domains, but not the C-terminal transmembrane helices, are already coiled up.

Many studies have attempted to reconstitute membrane fusion triggered by $\mathrm{Ca}^{2+}$-synaptotagmin-1 in vitro using SNARE-containing artificial membranes, either by adding the soluble cytoplasmic C2 domains (C2AB fragment; residues 97-421) or by inserting full-length membrane-anchored synaptotagmin-1. A major limitation of these studies is that SNAREs alone are sufficient to induce lipid mixing of liposomes ${ }^{23}$. Indeed, membrane fusion is relatively efficient when artificial membranes containing even a single copy ${ }^{24}$ of synaptobrevin-2 and a combination of syntaxin-1A and SNAP- 25 are mixed. The addition of the soluble $\mathrm{C} 2 \mathrm{AB}$ fragment makes membrane fusion somewhat $\mathrm{Ca}^{2+}$ sensitive and results in an increase in fusion efficiency between $100 \mu \mathrm{M}$ and $10 \mathrm{mM} \mathrm{Ca}^{2+}$ (mainly lipid mixing) $3,10,11,15,16,20,21,25-29$.

\footnotetext{
${ }^{1}$ Department of Neurobiology, Max Planck Institute for Biophysical Chemistry, Göttingen, Germany. ${ }^{2}$ Department of Dynamics of Complex Fluids, Max Planck Institute for Dynamics and Self-Organization, Göttingen, Germany. ${ }^{3}$ Department of Theoretical and Computational Biophysics, Max Planck Institute for Biophysical Chemistry, Göttingen, Germany. ${ }^{4}$ Institute for Organic and Biomolecular Chemistry, Georg-August University, Göttingen, Germany. ${ }^{5}$ Facility for Electron Microscopy, Max Planck Institute for Biophysical Chemistry, Göttingen, Germany. Correspondence should be addressed to R.J. (R.Jahn@gwdg.de).
} 
However, in these studies, membrane fusion still proceeds in the absence of the $\mathrm{C} 2 \mathrm{AB}$ fragment, and the increase in fusion efficiency is usually less than ten-fold, compared to the $>18,000$-fold increase in vivo ${ }^{30}$. Recently, it was reported that lipid mixing in the presence of membrane-anchored synaptotagmin-1 was increased about three- to five-fold at $10 \mu \mathrm{M} \mathrm{Ca}^{2+}$ but decreased again at higher $\mathrm{Ca}^{2+}$ concentrations ${ }^{27}$. This $\mathrm{Ca}^{2+}$ dependency was strongly influenced by the lipid composition of both liposome populations: increasing the fraction of anionic lipids in the Q-SNARE or R-SNARE membranes resulted in higher or lower $\mathrm{Ca}^{2+}$ sensitivity, respectively.

There is general consensus that the interactions between synaptotagmin-1 and membranes and SNAREs are predominantly electrostatic. Indeed, binding of synaptotagmin-1 both to anionic lipid membranes ${ }^{5,7}$ and to SNARE molecules ${ }^{18,19,22}$ is heavily influenced by the ionic strength of the solution. However, the influence of ionic interactions on membrane fusion in the presence of synaptotagmin-1 has not been investigated: all aforementioned in vitro synaptotagmin-1 membrane fusion studies were performed at relatively high, physiological ionic strength and typically $100-150 \mathrm{mM} \mathrm{KCl}$ or $\mathrm{NaCl}$. At these high salt concentrations, however, the binding of synaptotagmin-1 to SNAREs and anionic membranes is strongly reduced or even absent ${ }^{5,7,18,19,22}$. We thus set out to answer the question of how ionic strength influences the action of synaptotagmin-1.

\section{RESULTS}

\section{Membrane fusion is blocked at low ionic strength}

SNARE-mediated liposome fusion is typically studied at physiological ionic strength, with $\mathrm{NaCl}$ or $\mathrm{KCl}$ concentrations of $100-$ $150 \mathrm{mM}^{3,5,10,11,15,16,20,21,25-29}$ where the Debye charge screening length is only $\sim 7 \AA$. Molecules need to be within a few times this distance to interact. We studied SNARE-mediated fusion at low ionic strength by using a FRET-based lipid mixing assay (without synaptotagmin-1; Fig. 1a). The R-SNARE population of liposomes contained a 1:1,000 molar protein-to-lipid ratio of recombinant synaptobrevin-2 and was labeled with the fluorescent lipid analog Oregon greenphosphatidylethanolamine (OG-PE; donor fluorophore). The Q-SNARE population of liposomes contained 1:2,000 of a complex consisting of two copies of recombinant syntaxin-1A (lacking its inhibitory $\mathrm{N}$-terminal Habc domain ${ }^{31-33}$; residues $183-288$ ) and a single

Figure 1 Electrostatic repulsion blocks SNARE-mediated membrane fusion at low ionic strength. (a) Lipid mixing of DiD-labeled liposomes containing syntaxin-1A-SNAP-25 (Sx1A Sn25) with OG-PE-labeled liposomes containing synaptobrevin-2 (Sb2). Membrane fusion results in donor quenching. Fusion was measured at the $\mathrm{KCl}$ concentrations indicated. (b) As in a, but now for liposomes containing the synaptobrevin-2 49-96 -stabilized acceptor complex ${ }^{34}$. The osmolarity was preserved with sucrose. Lipid mixing was still blocked without sucrose (dashed green curve), but the liposomes were deformed by osmotic stress (inset, negative-staining EM; scale bar, $100 \mathrm{~nm}$ ). Synaptobrevin-2 $1-96$ inhibited fusion ( $R_{\text {sol }}$; for $150 \mathrm{mM} \mathrm{KCl}$ ). (c) As in $\mathbf{b}$, but now with pure phosphatidylcholine (PC) liposomes where lipid mixing occurred at both low (green) and normal (black) ionic strength. (d) Ten percent anionic phosphoserine (PS) blocked fusion at low, but not normal, ionic strength. (e) SNARE complex formation by FRET ${ }^{35}$. The soluble SNARE domain of synaptobrevin-2 (100 nM; $R_{\text {sol }}$; solid curves) or syntaxin-1A with SNAP-25 $\left(Q_{\text {sol }}\right.$; dashed curves) resulted in complex formation regardless of ionic strength. (f) By contrast, mixing liposomes containing membraneanchored synaptobrevin-2 and syntaxin-1A resulted in complex formation only at normal but not at low ionic strength regardless of $1 \mathrm{mM} \mathrm{Ca}^{2+}$ (pink). The structure ${ }^{58}$ shows the dye positions. The FRET efficiencies are underestimated because the $\sim 20 \%$ cross-talk is not accounted for. Typical curves of several repeats are shown. Experiments were performed with 4-8 nM liposomes at $20^{\circ} \mathrm{C}$.
SNAP-25 (2:1 complex $)^{25}$. Those liposomes were labeled with DiD (acceptor fluorophore). As already shown ${ }^{3,10,11,15,16,20,21,23-29,32-38}$, robust, SNARE-specific lipid mixing occurred at normal ionic strength (20 mM potassium HEPES, pH 7.4, $150 \mathrm{mM} \mathrm{KCl;} \mathrm{Fig.} \mathrm{1a).}$ However, lipid mixing was almost completely blocked at low ionic strength $(20 \mathrm{mM}$ potassium HEPES, pH 7.4, $5 \mathrm{mM} \mathrm{KCl,} 300 \mathrm{mM}$ sucrose) where the Debye screening length was $\sim 25 \AA$. Lipid mixing at low ionic strength was also blocked with a 1:8,000 molar protein-tolipid ratio of the binary Q-SNARE complex stabilized with a fragment of synaptobrevin-2 (residues 49-96; Fig. 1b). This stabilized complex ensures that all Q-SNAREs are available for fusion and shows markedly enhanced fusion efficiency compared to native Q-SNAREs ${ }^{34}$. These results indicate that the absence of lipid mixing at low ionic strength was not due to unavailability of the binary Q-SNARE complex. Reversing the fluorescent labels or increasing the SNARE density up to 1:500 yielded similar results.

We could not incorporate SNARE proteins in the liposomes at salt concentrations below $150 \mathrm{mM}$ and had to reduce the ionic strength by diluting the liposomes afterwards. We added sucrose to preserve the osmolarity at $\sim 340 \mathrm{mOsm}$. Membrane fusion was still blocked at low ionic strength when the sucrose was omitted, but the liposomes looked osmotically deformed by negative-staining electron microscopy (Fig. 1b), and the liposomes became leaky at osmotic differences above 100 mOsm (Supplementary Fig. 1a). The presence of sucrose increased the viscosity by $33 \%$, and this decreased the diffusion of both soluble and membrane-associated ${ }^{39}$ molecules. Diffusionlimited reactions were slowed accordingly ${ }^{40}$.
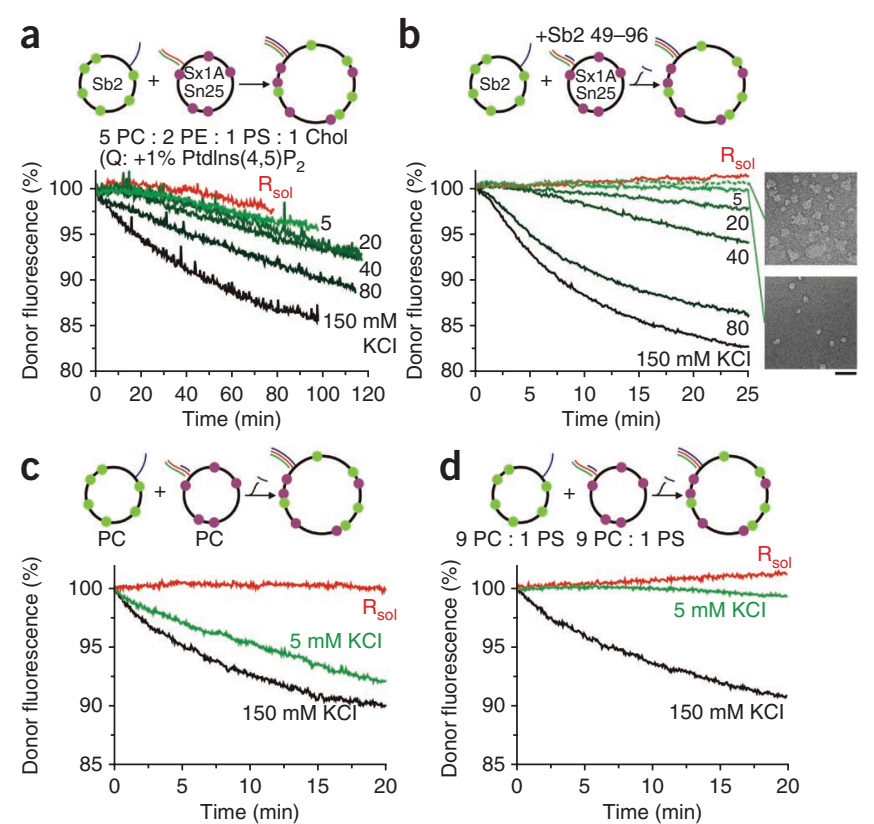

e

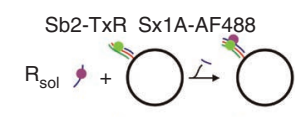

f Sb2-TxR Sx1A-AF488
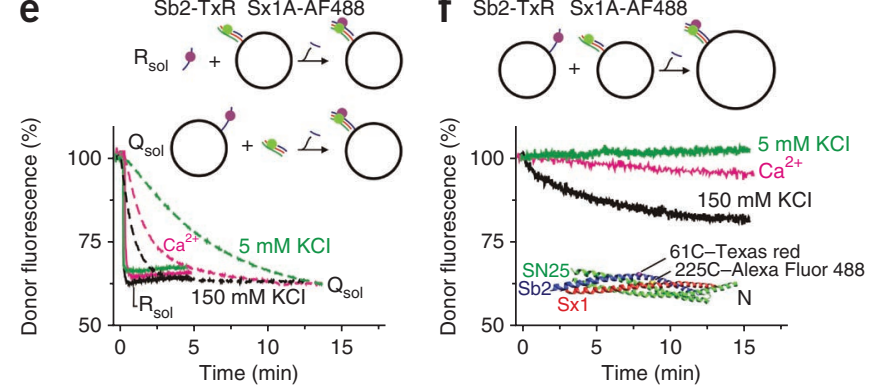
Figure 2 At low ionic strength, $\mathrm{Ca}^{2+}-$ synaptotagmin-1 triggers lipid mixing. (a,b) Lipid mixing of synaptobrevin-2synaptotagmin-1 liposomes with those containing syntaxin-1A-SNAP-25 (a) and the synaptobrevin249-96-stabilized acceptor complex ${ }^{34}$ (b). At low ionic strength, $1 \mathrm{mM}$

$\mathrm{Ca}^{2+}$ substantially increased lipid mixing. There was much less fusion with $1 \mathrm{mM}$ $\mathrm{Mg}^{2+}$. No lipid mixing was observed without synaptotagmin-1 (No Syt1) or synaptobrevin-2 (No Syb2). Synaptobrevin-2 ${ }_{1-96}\left(R_{\text {sol }}\right.$; for $1 \mathrm{mM} \mathrm{Ca}^{2+}$ ) or SNAP-25 and syntaxin-1 $\mathrm{A}_{183-262}$ $\left(Q_{\text {sol }}\right)$ inhibited fusion. (c-e) $\mathrm{Ca}^{2+}$-triggered lipid mixing measured by flow cytometry. (c) Donor and acceptor liposomes (as in a) were mixed in the $23 \mathrm{~cm} \times 100 \mu \mathrm{m} \times 80 \mu \mathrm{m}(\mathrm{LWH})$ flow chamber. The flow speed was kept constant. At $25 \%$ of the channel length, $\mathrm{Ca}^{2+}$ was introduced by fast focused mixing. (d,e) Acceptor fluorescence along the channel with fluorescence microscopy (d) and binning of the fluorescence (e), showing $\mathrm{Ca}^{2+}$-specific lipid mixing. (f) Lipid mixing at low ionic strength (as in a) at the $\mathrm{Ca}^{2+}$ concentrations indicated, with (solid) or without (dashed) $0.5 \mathrm{mM} \mathrm{Mg}^{2+}$. (g) As in $\mathbf{f}$, but now with the synaptobrevin-2 49-96-stabilized acceptor complex. (h) Fluorescence dequenching experiment showing $\mathrm{Ca}^{2+}$-dependent lipid mixing of synaptobrevin-2 $49-96^{-}$-stabilized acceptor complex liposomes with purified rat synaptic vesicles ${ }^{37}$. Experiments were performed with $4 \mathrm{nM}$ liposomes at $20^{\circ} \mathrm{C}$. Typical experiments from two to four independent repeats are shown.

The liposomes contained $\sim 10 \%$ anionic lipids, and we speculated that the electrostatic repulsion might be too high for SNAREmediated membrane fusion at low ionic strength. To validate this hypothesis, we performed experiments with liposomes composed of pure zwitterionic phospatidylcholine (Fig. 1c,d). Without phosphoserine, lipid mixing at low ionic strength still occurred, albeit somewhat more slowly than at normal ionic strength because of the sucrose (increased viscosity). By contrast, lipid mixing was completely blocked by $10 \%$ phosphoserine at low but not at normal ionic strength. Thus, the electrostatic repulsion at low ionic strength increases the energy barrier to membrane fusion. We then tested whether SNARE nucleation could still occur under these conditions.

We used FRET to follow SNARE complex formation directly with liposomes containing a 1:8,000 protein-to-lipid ratio of the stabilized Q-SNARE complex with syntaxin-1A labeled with Alexa Fluor 488 (S225C; donor fluorophore) $^{32,35}$. Mixing these liposomes with $100 \mathrm{nM}$ of the soluble synaptobrevin-2 ${ }_{1-96}$ fragment labeled with Texas red (S61C; acceptor fluorophore) ${ }^{35}$ resulted in fast complex formation independent of ionic strength (Fig. 1e). Accordingly, mixing liposomes containing full-length synaptobrevin-2 with a soluble Q-SNARE complex (SNAP-25, syntaxin-1 $\mathrm{A}_{183-262}$ and synaptobrevin-2 ${ }_{49-86}$ ) also resulted in complex formation, albeit more slowly. This decrease in the speed of complex formation is probably due to increased electrostatic repulsion at low ionic strength (the Q-SNARE complex has an acidic surface). In contrast to the soluble SNAREs, membraneanchored Q- and R-SNAREs formed complexes only at normal but not at low ionic strength (Fig. 1f), thus paralleling lipid mixing.
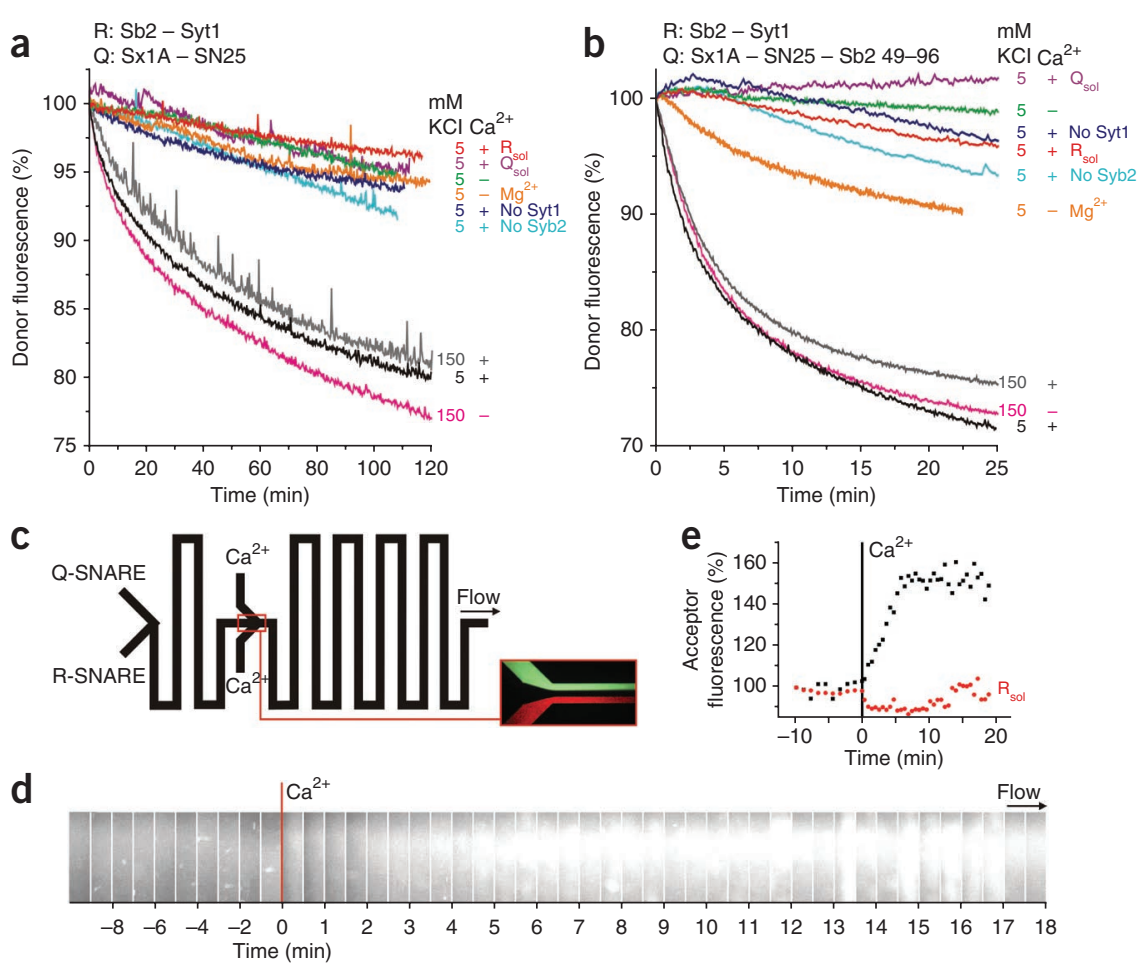

\section{$\stackrel{\text { low }}{\longrightarrow}$}
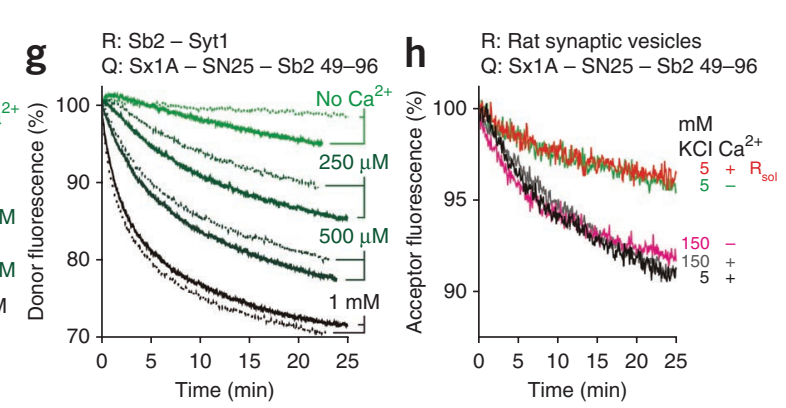

These FRET data agreed with the dissociation of the synaptobrevin$2_{49-96}$ fragment from the stabilized acceptor complex (Supplementary Fig. 1b,c). Thus, we conclude that although SNARE zippering can occur at low ionic strength, the high electrostatic repulsion between the liposomes blocks not only lipid mixing but also SNARE nucleation.

\section{$\mathrm{Ca}^{2+}$-synaptotagmin-1 action at low ionic strength}

Next, we investigated whether synaptotagmin-1 rescues membrane fusion at low ionic strength. We reconstituted recombinant full-length synaptotagmin-1 at a protein-to-lipid ratio of 1:4,000 in the R-SNARE liposomes. At normal ionic strength, synaptotagmin-1 increased the lipid mixing kinetics independently of $\mathrm{Ca}^{2+}$ (Fig. 2a,b), as reported previously ${ }^{25,29}$. By contrast, lipid mixing was almost completely blocked at low ionic strength; under these conditions, $1 \mathrm{mM} \mathrm{Ca}{ }^{2+}$ markedly increased lipid mixing. This lipid mixing depended strictly on both synaptotagmin-1 and the SNAREs. At low ionic strength, the C2 domains of synaptotagmin-1 are correctly folded and specifically bind $\mathrm{Ca}^{2+}$ (Supplementary Fig. 2). Experiments in which $\mathrm{Ca}^{2+}$ was mixed by controlled flow focusing in a microfluidics flow chamber ${ }^{41}$ showed that fusion could be readily triggered by $\mathrm{Ca}^{2+}$ (Fig. 2c-e). Compared to cuvette-based experiments, flow cytometry allows relatively fast and well-controlled mixing. Moreover, under our conditions, the synaptotagmin-1-mediated membrane fusion efficiency correlated with the $\mathrm{Ca}^{2+}$ concentration in a dose-dependent manner 
Figure $3 \mathrm{Ca}^{2+}$-synaptotagmin-1 triggers full membrane fusion at low ionic strength. (a) Content mixing ${ }^{24}$ with a self-quenching concentration of calcein encapsulated in liposomes with the stabilized Q-SNARE complex. Fusion with empty R-SNARE liposomes resulted in specific fluorescence dequenching. Synaptobrevin- $2_{1-96}\left(R_{\text {sol }}\right)$ or the absence of $\mathrm{Ca}^{2+}$ or synaptotagmin-1 (No Syt1) abolished fusion. (b) $\mathrm{Ca}^{2+}$ dependent SNARE complex formation by FRET as in Figure $1 \mathbf{f}$ with membraneanchored synaptobrevin-2-synaptotagmin-1. (c-e) SNARE complex formation with FRET. Liposomes containing the stabilized Q-SNARE complex were incubated with synaptobrevin-2 $1-116$ liposomes without $\mathrm{Ca}^{2+}$. The synaptobrevin-2 liposomes contained no synaptotagmin-1 (c), wildtype (WT) synaptotagmin-1 (d) or the KAKA mutant (e). At the indicated times, synaptobrevin-2 ${ }_{1-96}$ was added, either unlabeled (red curve; in case of Texas red-labeled synaptobrevin-2 1-116) or Texas red labeled (blue; in case of unlabeled synaptobrevin-2 1-116). Black curve, control

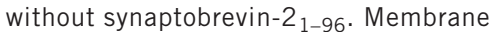
fusion was triggered with $\mathrm{Ca}^{2+}$. Synaptobrevin2 ${ }_{1-96}$ bound to the Q-SNAREs regardless of the presence of synaptobrevin-2 $1-116^{-}$ synaptotagmin-1 liposomes. (f) Lipid mixing by wild-type $\mathrm{Ca}^{2+}$-synaptotagmin-1 $(A B)$ and $\mathrm{Ca}^{2+}$-binding disruption mutants to $C 2 B\left(A b^{*}\right), C 2 A(a * B)$ or both $\left(a^{*} b^{*}\right)$. (g) SNARE complex formation by FRET for the synaptotagmin-1 mutants paralleled lipid mixing. (h) Lipid mixing of synaptobrevin-2synaptotagmin-1 liposomes with $10-20-\mu \mathrm{m}$ GUVs containing the Q-SNAREs (inset, fluorescence microscopy; scale bar, $50 \mu \mathrm{m}$ ). Disrupting binding of $\mathrm{Ca}^{2+}$ to the $\mathrm{C} 2 \mathrm{~B}$ domain abolished fusion with GUVs. We used $\sim 4 \mathrm{nM}$ liposomes at $20^{\circ} \mathrm{C}$. Typical curves from two or three independent repeats are shown.

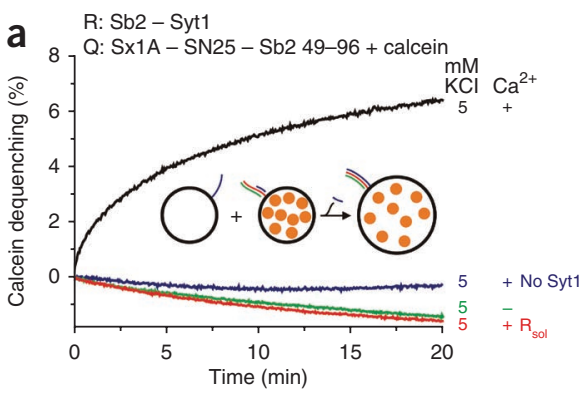

b $\quad R: S b 2-T x R-S y t 1$
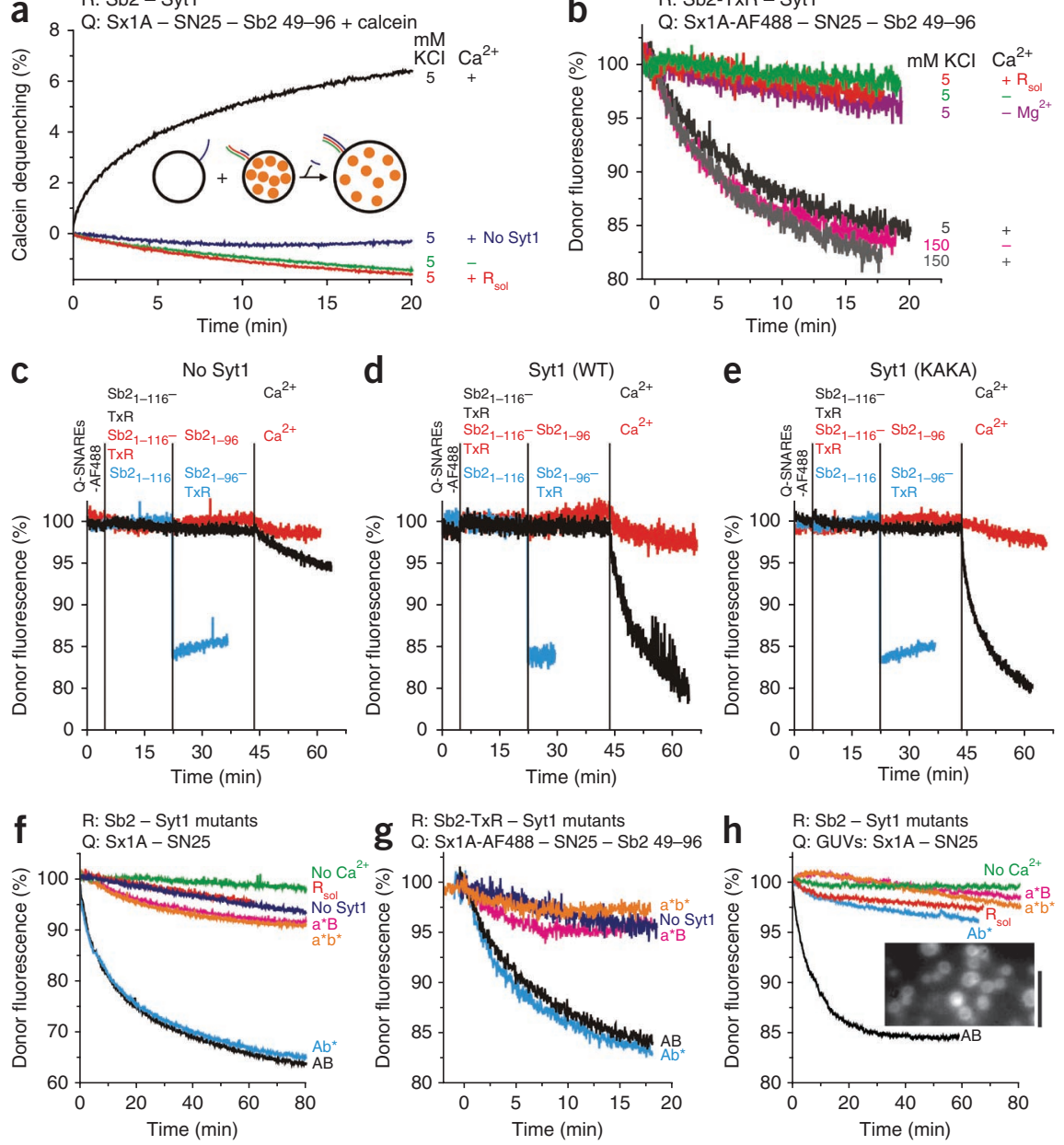

from $10 \mu \mathrm{M}$ up to $1 \mathrm{mM} \mathrm{Ca}^{2+}$, and $\mathrm{Mg}^{2+}$ had little effect (Fig. $2 \mathbf{f}-\mathbf{g}$ ). This $\mathrm{Ca}^{2+}$ sensitivity depends on precise protein densities and compositions of the membranes and buffer ${ }^{27}$.

At normal ionic strength, purified synaptic vesicles from rat brain fuse independently of $\mathrm{Ca}^{2+}$ (ref. 37). However, as these membranes contain about $15 \%$ anionic lipids ${ }^{42}$, it seemed possible that (much as for the liposomes) membrane fusion of synaptic vesicles becomes $\mathrm{Ca}^{2+}$-sensitive at low ionic strength. To test this hypothesis, we fused synaptic vesicles with Q-SNARE liposomes containing both donor and acceptor fluorophores. In this case, membrane fusion dilutes the fluorescent lipid analogs with the vesicle membranes, resulting in a loss of FRET and a decrease in acceptor fluorescence. At low ionic strength, lipid mixing was largely $\mathrm{Ca}^{2+}$ dependent (Fig. 2h). Thus, at low ionic strength $\mathrm{Ca}^{2+}$-synaptotagmin-1 triggers lipid mixing not only of liposomes, but also of native synaptic vesicles.

Because lipid mixing does not distinguish between hemifusion and full membrane fusion, it was conceivable that the membrane fusion did not progress beyond the hemifusion state. To resolve this issue, we used a content mixing assay in which liposomes with encapsulated calcein at self-quenching concentrations were fused with empty (calcein-free) liposomes ${ }^{24}$. Content mixing results in calcein dequenching. The assay showed an increase in fluorescence that was specific to SNARE and $\mathrm{Ca}^{2+}$-synaptotagmin-1 (Fig. 3a). This content mixing was not caused by leakage of the calcein from the liposomes, as leakage was only $4-5 \%$ of total calcein (Supplementary Fig. 3a). In addition, FRET and fluorescence anisotropy measurements indicated that there was no SNARE nucleation without $\mathrm{Ca}^{2+}$ (Figs. 1e,f, 3b-e). In the absence of synaptotagmin-1, there was some residual SNARE complex formation (Figs. 1e and 3c). We attribute this to charge-shielding by the bivalent $\mathrm{Ca}^{2+}$ cations, which allows a low degree of SNARE interaction and membrane fusion. The formation of SNARE complexes was inhibited by synaptobrevin- $2_{1-96}$ even when it was added after the mixing of the R- and Q-SNARE liposomes, and regardless of whether we used wild-type synaptotagmin-1 or a mutant in which two lysines of the polybasic patch are converted to alanines (K325A K326A; KAKA mutant ${ }^{7}$; Fig. 3d,e). This indicates that there was no substantial SNARE nucleation without $\mathrm{Ca}^{2+}$.

Disrupting the ability of the C2A domain to bind $\mathrm{Ca}^{2+}$ (D178A D230A D232A) reduced both lipid mixing and formation of SNARE complexes substantially (Fig. 3f,g). By contrast, the corresponding C2B mutation (D309A D363A D365A) had little effect on membrane fusion efficiency. This has been reported before ${ }^{11,16,25}$, but it contradicts in vivo findings that disruption of $\mathrm{Ca}^{2+}$ binding to the $\mathrm{C} 2 \mathrm{~B}$ domain impaired release much more severely than C2A disruption ${ }^{43}$. This discrepancy may exist because-unlike in the synapseboth fusion partners are small liposomes, and this bypasses the requirement for membrane bending by the $\mathrm{C} 2 \mathrm{~B}$ domain ${ }^{15,16}$. 
a

$$
\frac{\text { Syt1 } 1-421}{--++} \mathrm{Ca}^{2+}
$$

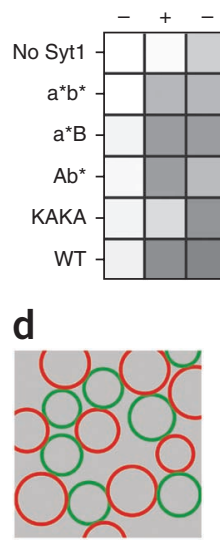

e

To evaluate whether membrane curvature influences the ability of the $\mathrm{C} 2 \mathrm{~B}$ domain to stimulate membrane fusion at low ionic strength, we performed experiments with $10-20-\mu \mathrm{m}$ giant unilamellar vesicles (GUVs; Fig. 3h). These membranes are much less curved than the small 36-nm liposomes ${ }^{24}$ used elsewhere in this study. Fusing the GUVs with small synaptotagmin-1-synaptobrevin-2 liposomes showed that (much like normal ionic strength) disruption of $\mathrm{Ca}^{2+}$ binding to the $\mathrm{C} 2 \mathrm{~B}$ mutant abolished fusion ${ }^{16}$. Fusion of the $\mathrm{C} 2 \mathrm{~B}$ mutant could be rescued through disruption of the GUVs by sonication (Supplementary Fig. 3b). We conclude that at low ionic strength $\mathrm{Ca}^{2+}$-synaptotagmin-1 triggers SNARE-mediated membrane fusion and that both $\mathrm{C} 2$ domains are required.

\section{Synaptotagmin-1 acts as a distance regulator}

Synaptotagmin-1 binds to anionic lipids both with and without $\mathrm{Ca}^{2+}$, and this binding leads to membrane clustering ${ }^{5,7-13,25}$. Negative-staining EM showed strong instantaneous clustering of the liposomes when synaptotagmin-1 liposomes were mixed with empty liposomes (no SNAREs; Supplementary Fig. 4a). This clustering was specific to $\operatorname{PtdIns}(4,5) \mathrm{P}_{2}$ and synaptotagmin- 1 and was reduced when we used the KAKA mutant of synaptotagmin-1 (Supplementary Fig. 4b), as also reported for normal ionic strength ${ }^{9}$. We obtained identical results when the SNAREs were present. The clustering of liposomes by synaptotagmin-1 was confirmed with dynamic light scattering (DLS) experiments (Fig. 4a and Supplementary Fig. 4c). In addition, we developed a microscale thermophoresis ${ }^{44}$ assay to assess liposome clustering without $\mathrm{Ca}^{2+}$ (Fig. 4b,c). We inserted DiD-labeled liposomes into a capillary that was heated by $\sim 5^{\circ} \mathrm{C}$ locally with a focused

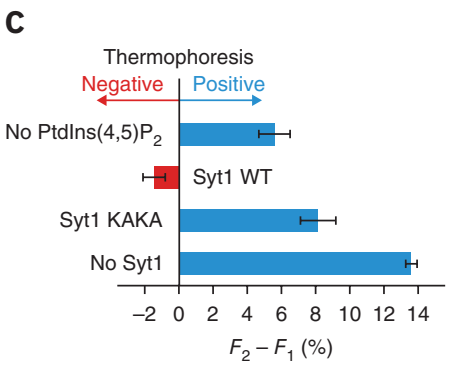

h
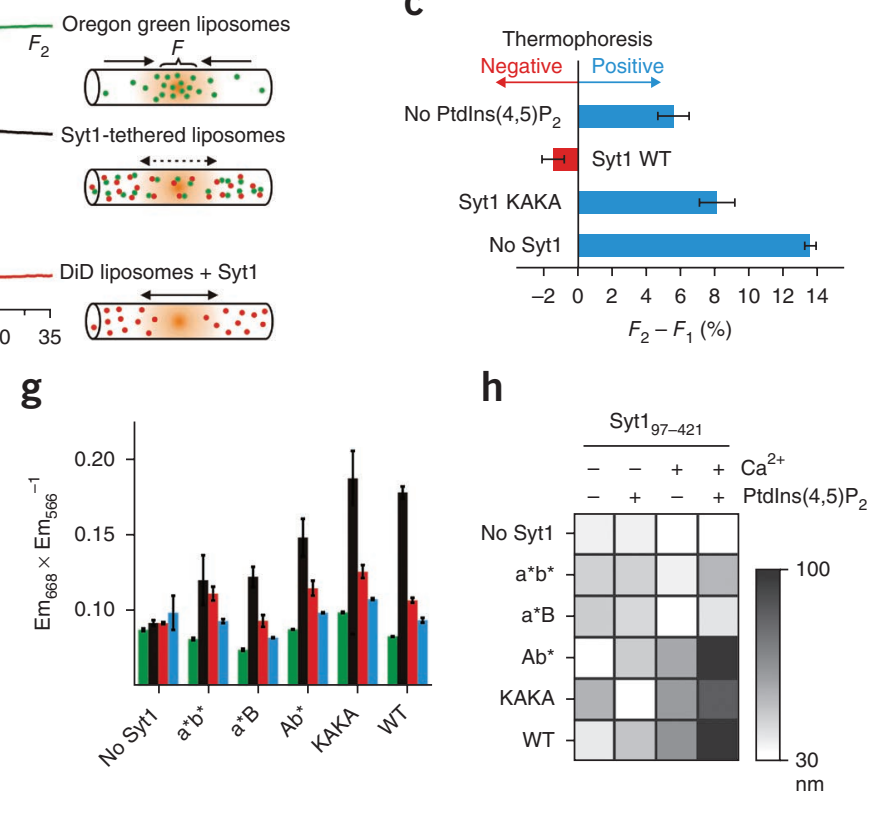

Figure 4 Liposome clustering by synaptotagmin-1. (a) DLS at low ionic strength. Liposomes with 1:4,000 synaptotagmin-1 mutants were mixed 1:1 with PtdIns(4,5)P2 liposomes (no SNAREs; bar graphs and polydispersities, Supplementary Fig. 4c). The grayscale indicates the liposome cluster size. (b) Microscale capillary thermophoresis 44,45 tethering experiment. OG-PE-labeled liposomes move toward the heated spot in a capillary (negative Tethering was reduced without PtdIns(4,5)P2 or with the KAKA mutant. (d,e) Docking arrangements with liposomes tethered close (d) or further away (e). (f) FRT docking 46 immediately after mixing Dil-liposomes containing 1:4,000 synaptotagmin-1 with DiD-liposomes. No FRET was observed without Ca ${ }^{2+}$ reduced without PtdIns(4,5)P2. (g) The acceptor over donor fluorescence from $f( \pm s . d ., n=3$ ). (h) DLS as in a, but with 10 nM of the C2AB fragment (see also Supplementary Fig. 4f). Total liposome concentrations were 2-4 nM. Typical data from two or three independent repeats are shown.

infrared laser. Because of the Soret effect ${ }^{45}$, these liposomes thermodiffused away from the heated spot, thereby creating a local drop in concentration and fluorescence intensity (positive thermophoresis). The extent of this depletion depends on the surface properties of the liposomes (solvation entropy). In contrast to the DiD liposomes, liposomes labeled with sufficient ( $>5 \mathrm{~mol} \%$ ) OG-PE thermodiffuse toward the heated spot and are locally enriched (negative thermophoresis). Thus, DiD liposomes were separated from OG-PE liposomes by the heating laser unless they were tethered by synaptotagmin- 1 , in which case they co-segregated, resulting in intermediate thermophoresis. This tethering depended on $\operatorname{PtdIns}(4,5) \mathrm{P}_{2}$ and was reduced when we used the KAKA mutant of synaptotagmin-1, consistent with the DLS and EM data. Density gradient flotation experiments with various mutants of the soluble $\mathrm{C} 2 \mathrm{AB}$ domain showed that without $\mathrm{Ca}^{2+}$, the polybasic lysine patch of synaptotagmin- 1 bound to membranes, whereas binding with $\mathrm{Ca}^{2+}$ occurred mainly at the $\mathrm{Ca}^{2+}$ binding sites $^{7,9}$ (Supplementary Fig. 4d).

In the EM images, synaptotagmin-1 tethered the liposomes at very narrow distances even without $\mathrm{Ca}^{2+}$ (so that they appear 'squeezed together'; Supplementary Fig. 4a and Fig. 4d). Regardless of this tight liposome association, SNARE nucleation did not occur under those conditions (Fig. $\mathbf{3 b}-\mathbf{e}$ ), which suggests that the vesicles were still separated by a distance too far for trans SNARE interactions (Fig. 4e). However, negative-staining EM involves fixation and drying and is therefore unsuitable for assessing membrane distances. We could not perform cryo-EM, because of the presence of sucrose to compensate for the osmolarity. Therefore, we used a FRET approach ${ }^{46}$ (Fig. 4f,g and Supplementary Fig. 4 e), based on the $\sim 5 \%$ FRET that results 

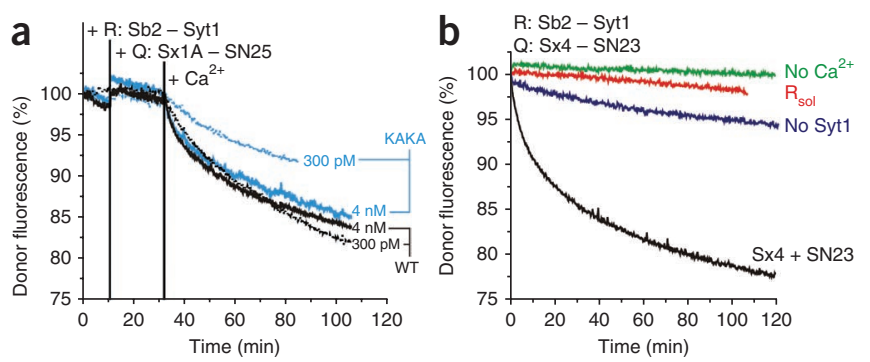

Figure 5 Preclustering of liposomes accelerates lipid mixing. (a) We incubated $150 \mathrm{pM}$ or $2 \mathrm{nM}$ of R-SNARE liposomes for 20 min with the same amounts of Q-SNARE liposomes. Subsequently, lipid mixing was triggered by addition of $\mathrm{Ca}^{2+}$. For the high ( $4 \mathrm{nM}$ total) liposome concentrations, the lipid mixing efficiency of wild-type synaptotagmin-1 was comparable to that of the KAKA mutant. By contrast, for the low liposome concentrations ( $300 \mathrm{pM}$ ), the fusion efficiency was substantially lower for the KAKA mutant compared to the wild type. (b) Lipid mixing of full-length synaptotagmin-1-synaptobrevin-2 liposomes with liposomes containing the Q-SNAREs of constitutive exocytosis (syntaxin-4 and SNAP-23). We saw no fusion in the absence of $\mathrm{Ca}^{2+}\left(\mathrm{No} \mathrm{Ca}^{2+}\right)$, and fusion could be blocked with synaptobrevin-2 ${ }_{1-96}$ $\left(R_{\text {sol }}\right)$. All fusion experiments were performed at $20{ }^{\circ} \mathrm{C}$. Typical curves from two independent repeats are shown.

from clustering of DiI with DiD-labeled liposomes at distances below $\sim 5 \mathrm{~nm}$. In the presence of synaptotagmin-1 (no SNAREs), we detected no FRET signal although the vesicles were clustered (Fig. $\mathbf{4 a}-\mathbf{c}$ and Supplementary Fig. 4a-d). Thus, $\mathrm{Ca}^{2+}$ induced FRET of both the wild-type and the KAKA mutant, and this increased substantially when $1 \% \operatorname{PtdIns}(4,5) \mathrm{P}_{2}$ was present. Disrupting binding of $\mathrm{Ca}^{2+}$ to the $\mathrm{C} 2 \mathrm{~A}$ or $\mathrm{C} 2 \mathrm{~B}$ domain largely impaired this FRET signal. The observed FRET was caused by close membrane proximity rather than by residual lipid mixing, because the FRET signal was reversed by addition of $1 \mathrm{mM}$ EDTA. Finally, DLS experiments with the C2AB fragment (Fig. 4h and Supplementary Fig. 4f) showed that this fragment was sufficient for liposome clustering in the presence of $\mathrm{Ca}^{2+}$, as also reported for normal ionic strength ${ }^{9}$.

We conclude that without $\mathrm{Ca}^{2+}$, the polybasic patch of synaptotagmin-1 tethers the liposomes too far apart for SNARE nucleation. $\mathrm{Ca}^{2+}$ binds to the $\mathrm{C} 2 \mathrm{AB}$ domain, which functions as a charge bridge' to bring the membranes close enough for SNARE nucleation. A comparison of wild-type synaptotagmin- 1 and the KAKA mutant (with reduced membrane tethering) indicates that this tethering can

Figure 6 Models of distance regulation by synaptotagmin-1. (a) Molecular dynamic simulations to estimate the maximal distances between the various domains. In the simulations, we pulled pairwise on various membranebinding sites of the $C 2 A B$ domain: the $N$ terminus, the polybasic lysine patch (KKKK) and the $\mathrm{Ca}^{2+}$-binding sites of the $\mathrm{C} 2 \mathrm{~A}$ and $\mathrm{C} 2 \mathrm{~B}$ domains. The two conserved arginines (R398 and R388) are shown in brown; the linker is in black. The $\mathrm{N}$ terminus is connected to the transmembrane helix with a 61-residue linker that can extent to $\sim 23 \mathrm{~nm}$. Maximal distances are indicated; these are approximate for the tethering distances of the membranes but do not take into account additional interactions such as membrane insertion and bending. (b) Model of synaptotagmin-1-mediated lipid mixing. In the absence of $\mathrm{Ca}^{2+}$, synaptotagmin-1 (gray) tethers to anionic membranes, and particularly to Ptdlns(4,5)P2 (orange) through its polybasic lysine patch. The distance is too far for SNAREs to form complexes (synaptobrevin-2: blue; syntaxin-1A: red; SNAP-25: green). Step $\mathrm{A}$ : in the presence of $\mathrm{Ca}^{2+}$ (purple), a transitional conformation change occurs and synaptotagmin- 1 binds the membrane by its calcium binding pockets and basic residues on the $\mathrm{C} 2 \mathrm{AB}$ domain ${ }^{9}$. This drives the membranes together and SNARE complex formation can occur. Step B: the formation of SNARE complexes drives membrane fusion. accelerate lipid mixing at low, rate-limiting concentrations of liposomes (Fig. 5a), but not at higher concentrations (Supplementary Fig. 5a), in agreement with the weak phenotype of the KAKA mutant ${ }^{7,47}$. A specific interaction between synaptotagmin-1 and the neuronal SNAREs does not seem to be required, because the SNAREs of constitutive exocytosis (SNAP-23 and syntaxin-4 without Habc domain; residues 191-298) can also mediate membrane fusion triggered by $\mathrm{Ca}^{2+}$-synaptotagmin-1 (Fig. 5b). These findings are not surprising because $\mathrm{Ca}^{2+}$-synaptotagmin-1 is not required to interact with SNAREs in order to bring two membranes close together ${ }^{9}$ (Fig. 4). However, this contradicts earlier data on the $\mathrm{C} 2 \mathrm{AB}$ fragment ${ }^{11,25}$, probably because (i) these studies were performed with much higher SNARE densities and at normal ionic strength where synaptotagmin-1-SNARE interactions may help to tether the liposomes ${ }^{25}$ and might modulate the SNAREs ${ }^{3,9,11,15,16,18-22}$; and (ii) we cannot exclude the possibility that synaptotagmin-1 interacts with SNAP-23-syntaxin-4 at low ionic strength. Last, lipid-mixing experiments showed that liposome tethering at a close distance by the $\mathrm{Ca}^{2+}$-bound $\mathrm{C} 2 \mathrm{AB}$ fragment is sufficient to trigger membrane fusion (Supplementary Fig. 5b), similar to normal ionic strength $3,10,11,15,16,20,25-29$.

We estimated how far apart synaptotagmin-1 could tether two membranes in various conformations with coarse-grain molecular dynamics simulations ${ }^{48,49}$. The $\mathrm{C} 2 \mathrm{AB}$ domain was simulated on the basis of its crystal structure (PDB 1DQV $)^{50}$. Each individual C2 domain was conformationally fixed but had full translational and rotational mobility and was connected by a flexible linker (residues 266-273), as supported by NMR data ${ }^{9,18}$. We then pulled pairwise on the $\mathrm{Ca}^{2+}$-binding patches, the polybasic patch and the $\mathrm{N}$ terminus (Fig. 6a). The distances between these sites reflect the maximum distance at which synaptotagmin- 1 can connect two membranes and are determined by the interconnecting linker and the surface interactions between the $\mathrm{C} 2$ domains. These distances are overestimates,
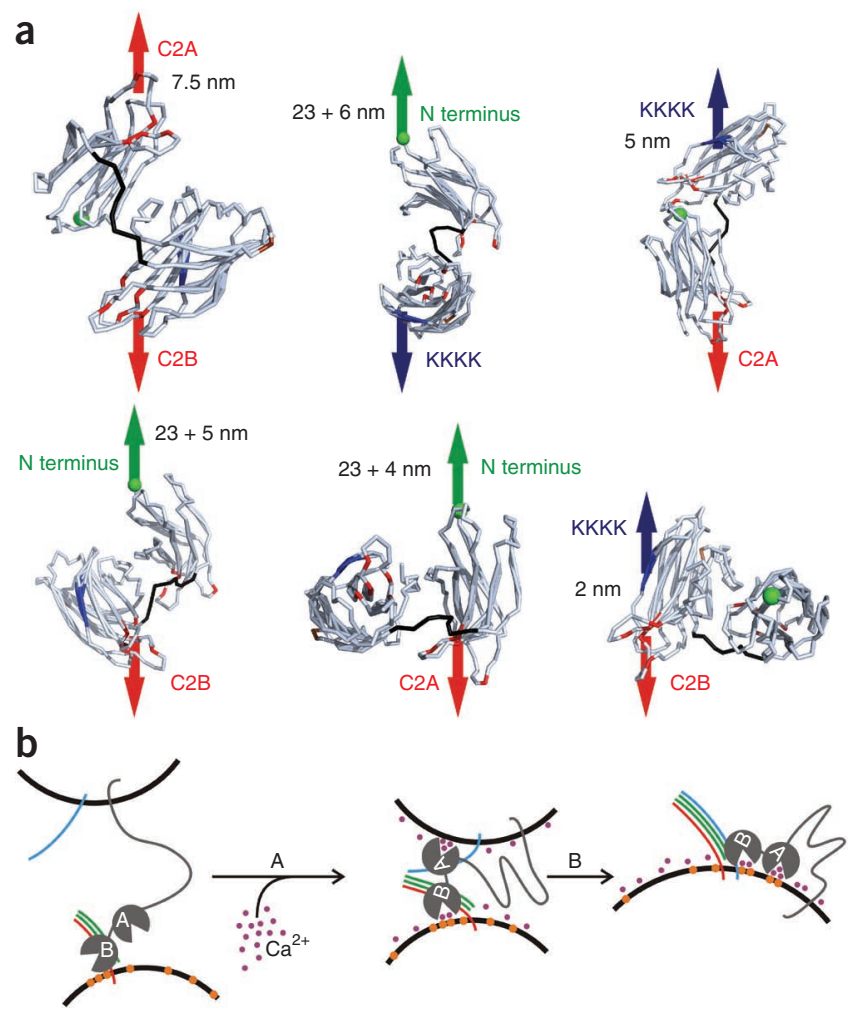
because membrane insertion and bending are not accounted for. The distance from the polybasic patch to the transmembrane helix is $\sim 28 \mathrm{~nm}$ (including the $\sim 23 \mathrm{~nm}$ linker). However, the distance at which synaptotagmin-1 tethers liposomes without $\mathrm{Ca}^{2+}$ is probably shorter than that (as the Debye length is only $\sim 25 \AA$ ), but higher than the $\sim 5 \mathrm{~nm}$ determined from the FRET experiments ${ }^{46}$ (Fig. 4f,g). The maximum distance across which the $\mathrm{Ca}^{2+}$-bound $\mathrm{C} 2 \mathrm{AB}$ domain could span two membranes is $\sim 2-7.5 \mathrm{~nm}$, close to the $4 \mathrm{~nm}$ determined from cryo$\mathrm{EM}^{9}$. These distances would explain why decreasing the ionic strength and increasing the Debye length from $\sim 7$ to $25 \AA$ has such a marked effect on membrane fusion. Thus, at low ionic strength, $\mathrm{Ca}^{2+}$ changes the membrane distance from $5-28 \mathrm{~nm}$ to below that required for SNARE complex formation ( $8 \mathrm{~nm}$ at normal ionic strength ${ }^{36}$ ) so that membrane fusion can occur.

\section{DISCUSSION}

At low ionic strength, synaptotagmin-1 can regulate membrane fusion upstream of SNARE nucleation by acting as a distance regulator. In the absence of $\mathrm{Ca}^{2+}$, the polybasic patch on the $\mathrm{C} 2 \mathrm{~B}$ domain tethers the liposomes, but electrostatic repulsion keeps them too far apart for SNARE complexes to form (Fig. 6b; step A). The linker that connects the $\mathrm{C} 2 \mathrm{AB}$ domain to the vesicle membrane is 61 residues long (residues 81-142), which means that it can extend to a maximum of $\sim 23 \mathrm{~nm}(0.38 \mathrm{~nm}$ per residue) - a distance longer than the $\sim 2 \times 25 \AA$ electrical double layers that separate the membranes. $\mathrm{Ca}^{2+}$ acts as a charge bridge, and the $\mathrm{Ca}^{2+}$-bound $\mathrm{C} 2 \mathrm{AB}$ domain docks the vesicles closer together, where SNARE complex formation and membrane fusion can occur (step B). Similar models in which synaptotagmin-1 functions as a membrane tethering factor have been proposed ${ }^{9,21,51}$, and we now present direct evidence that, at least at low ionic strength, such a mechanism can trigger membrane fusion.

We needed low ionic strength to ensure that the membrane repulsion was large enough to prevent SNARE nucleation. Although this condition does not reflect physiological conditions, we isolated an intermediate state at which vesicles can be tethered (for example, by synaptotagmin-1) but the distance between the membranes was too high for synaptotagmin-1-independent membrane fusion and synaptotagmin-1-mediated reworking of the SNAREs and the membranes ${ }^{3,9,11,15-22}$. Binding of $\mathrm{Ca}^{2+}$ to synaptotagmin-1 reduced the distance between the docked membranes, bringing them close enough for SNARE nucleation and subsequent fusion. Although specific increases in membrane fusion efficiency have been reported previously for both the C2AB fragment ${ }^{3,10,11,15,16,20,21,25-29}$ and full-length synaptotagmin-1 (ref. 27), this increase is larger in our case because 'background' fusion (in the absence of synaptotagmin-1 or $\mathrm{Ca}^{2+}$ ) is essentially blocked by keeping the membranes apart at low ionic strength. Thus, our finding adds a new facet to the controversially discussed mechanism of synaptotagmin-1 action in exocytosis and may help researchers to interpret many of the often conflicting data on synaptotagmin-1-mediated fusion of liposomes ${ }^{3,10,11,15,16,20,25-29}$. The question is how this mechanism is integrated in the molecular steps that govern vesicle docking and fusion in the synapse. In our opinion, two alternative scenarios are possible: one 'mainstream' scenario in which synaptotagmin-1 acts downstream of SNARE nucleation (which does not preclude an additional earlier role in docking ${ }^{52}$ ), and an alternative scenario according to which SNAREs are not in trans-contact with each other before synaptotagmin-1 receives the $\mathrm{Ca}^{2+}$ signal.

In the first case, docked and primed synaptic vesicles are characterized by the SNAREs being partially zippered in trans, as depicted in almost all current models ${ }^{1-4}$. Further zippering is prevented by an energy barrier that may involve membrane straining or inhibitory proteins such as complexins (or both). Indeed, mutants that interfered with either $\mathrm{N}$-terminal or C-terminal SNARE zippering had profoundly different effects on exocytosis in chromaffin cells, which can best be explained by kinetically separate states (docking and fusion) ${ }^{53}$. Accordingly, the ability of synaptotagmins to pull membranes a bit closer together may contribute to the overcoming of the energy barrier by relieving strain, in addition to an increase in local curvature or an activation or disinhibition of C-terminal SNARE zippering 3,9,11,15-22.

In the second case, $\mathrm{Ca}^{2+}$-synaptotagmin-1 would trigger membrane fusion by rapidly decreasing the distance between the vesicles and the plasma membrane, thereby allowing SNARE nucleation to occur (as proposed previously ${ }^{51}$ ). Thus, SNARE nucleation takes place after $\mathrm{Ca}^{2+}$ influx, consistent with our findings (Fig. 6b). It is unclear whether docked vesicles have their SNARE complexes arrested in a partially assembled complex ${ }^{53}$. There are good reasons for challenging the view that a metastable trans SNARE complex represents an energy minimum along the fusion pathway. First, knocking out synaptobrevin-2 (refs. 54,55) or cleaving it with tetanus neurotoxin ${ }^{56}$ does not affect vesicle docking, which is inconsistent with a requirement of SNARE nucleation for docking. Second, it is difficult to understand how a strained and partially zippered SNARE complex can be arrested, especially considering that only 1-3 SNARE complexes are required for membrane fusion ${ }^{24,57}$. At least in vitro, even the synaptobrevin- $2_{49-96}$ fragment (which binds with nanomolar affinity to the Q-SNAREs) cannot block membrane fusion ${ }^{34}$. Although both candidates for such a clamping role, synaptotagmin-1 (ref. 26) and complexin $^{20,22}$, can reduce membrane fusion, they bind with much lower affinity to the Q-SNARE complex than to synaptobrevin-2 $49-96$. Although effective energies may be different at the fusion site, there is no strong and direct evidence that any of these proteins slow or prevent SNARE assembly.

In summary, our data show that in a simple reconstituted system, key elements of synaptic transmission can be mimicked if SNARE nucleation is prevented by keeping the membranes apart before $\mathrm{Ca}^{2+}$ triggering. Under these conditions, no fusion occurs even though SNAREs are fully active, but fusion is 'unleashed' as soon as the membranes are pulled a bit closer by $\mathrm{Ca}^{2+}$-dependent membrane binding of the C2 domains (Fig. 6b). Although we needed to use a non-physiological trick (low ionic strength) to keep the membranes apart, it is conceivable that this separator role is fulfilled by proteins that reside in the space between the vesicle and the plasma membrane. Considering that both synaptic vesicles and release sites are crowded with membrane proteins ${ }^{42}$, such a scenario is not unlikely. However, further experiments are needed to determine whether, under physiological conditions, SNARE nucleation occurs before or after $\mathrm{Ca}^{2+}$ triggering in regulated exocytosis of synaptic vesicles.

\section{METHODS}

Methods and any associated references are available in the online version of the paper at http://www.nature.com/nsmb/.

Note: Supplementary information is available on the Nature Structural \& Molecular Biology website.

\section{ACKNOWLEDGMENTS}

We thank A. Stein and U. Ries for protein purification and comments. G.v.d.B. is financed by the Human Frontier Science Program. This work was supported by the US National Institutes of Health (P01 GM072694 to R.J.) and the Deutsche Forschungsgemeinschaft (SFB755 to S.T. and S.H.; SFB803 to K.M., J.H.R., M.H., U.D., H.G. and R.J.)

\section{AUTHOR CONTRIBUTIONS}

S.T. and S.H. performed the flow cytometry experiments. J.H.R. and

H.G. performed the MD simulations. M.H. purified the synaptic vesicles.

Thermophoresis data were from K.M. and U.D. D.R. performed the EM. G.v.d.B. 
performed all other experiments. G.v.d.B. and R.J. designed the study and wrote the paper. All authors discussed the results and commented on the manuscript.

\section{COMPETING FINANCIAL INTERESTS}

The authors declare no competing financial interests.

Published online at http://www.nature.com/nsmb/.

Reprints and permissions information is available online at http://www.nature.com/ reprints/index.html.

1. Brunger, A.T., Weninger, K., Bowen, M. \& Chu, S. Single-molecule studies of the neuronal SNARE fusion machinery. Annu. Rev. Biochem. 78, 903-928 (2009).

2. Jahn, R. \& Scheller, R.H. SNAREs - engines for membrane fusion. Nat. Rev. Mol. Cell Biol. 7, 631-643 (2006).

3. Chapman, E.R. How does synaptotagmin trigger neurotransmitter release? Annu. Rev. Biochem. 77, 615-641 (2008).

4. Martens, S. \& McMahon, H.T. Mechanisms of membrane fusion: disparate players and common principles. Nat. Rev. Mol. Cell Biol. 9, 543-556 (2008).

5. Radhakrishnan, A., Stein, A., Jahn, R. \& Fasshauer, D. The $\mathrm{Ca}^{2+}$ affinity of synaptotagmin 1 is markedly increased by a specific interaction of its $\mathrm{C} 2 \mathrm{~B}$ domain with phosphatidylinositol 4,5-bisphosphate. J. Biol. Chem. 284, 25749-25760 (2009).

6. Fernandez, I. et al. Three-dimensional structure of the synaptotagmin $1 \mathrm{C} 2 \mathrm{~B}-$ domain: synaptotagmin 1 as a phospholipid binding machine. Neuron 32, 1057-1069 (2001).

7. Li, L. et al. Phosphatidylinositol phosphates as co-activators of $\mathrm{Ca}^{2+}$ binding to $\mathrm{C} 2$ domains of synaptotagmin 1. J. Biol. Chem. 281, 15845-15852 (2006).

8. Bai, J., Tucker, W.C. \& Chapman, E.R. PIP2 increases the speed of response of synaptotagmin and steers its membrane-penetration activity toward the plasma membrane. Nat. Struct. Mol. Biol. 11, 36-44 (2004).

9. Araç, D. et al. Close membrane-membrane proximity induced by $\mathrm{Ca}^{2+-d e p e n d e n t}$ multivalent binding of synaptotagmin-1 to phospholipids. Nat. Struct. Mol. Biol. 13, 209-217 (2006)

10. Gaffaney, J.D., Dunning, F.M., Wang, Z., Hui, E. \& Chapman, E.R. Synaptotagmin $\mathrm{C} 2 \mathrm{~B}$ domain regulates $\mathrm{Ca}^{2+}$-triggered fusion in vitro: critical residues revealed by scanning alanine mutagenesis. J. Biol. Chem. 283, 31763-31775 (2008).

11. Bhalla, A., Chicka, M.C., Tucker, W.C. \& Chapman, E.R. $\mathrm{Ca}^{2+}$-synaptotagmin directly regulates t-SNARE function during reconstituted membrane fusion. Nat. Struct. Mol. Biol. 13, 323-330 (2006)

12. Herrick, D.Z., Sterbling, S., Rasch, K.A., Hinderliter, A. \& Cafiso, D.S. Position of synaptotagmin I at the membrane interface: cooperative interactions of tandem C2 domains. Biochemistry 45, 9668-9674 (2006).

13. Hui, E., Bai, J. \& Chapman, E.R. Ca ${ }^{2+}$-triggered simultaneous membrane penetration of the tandem C2-domains of synaptotagmin I. Biophys. J. 91, 1767-1777 (2008).

14. Schiavo, G., Gu, Q.M., Prestwich, G.D., Söllner, T.H. \& Rothman, J.E. Calciumdependent switching of the specificity of phosphoinositide binding to synaptotagmin. Proc. Natl. Acad. Sci. USA 93, 13327-13332 (1996).

15. Martens, S., Kozlov, M.M. \& McMahon, H.T. How synaptotagmin promotes membrane fusion. Science 316, 1205-1208 (2007).

16. Hui, E., Johnson, C.P., Yao, J., Dunning, F.M. \& Chapman, E.R. Synaptotagminmediated bending of the target membrane is a critical step in $\mathrm{Ca}^{2+}$-regulated fusion. Cell 138, 709-721 (2009).

17. Bai, J., Wang, C.T., Richards, D.A., Jackson, M.B. \& Chapman, E.R. Fusion pore dynamics are regulated by synaptotagmint-SNARE interactions. Neuron 41, 929-942 (2004).

18. Vrljic, M. et al. Molecular mechanism of the synaptotagmin-SNARE interaction in $\mathrm{Ca}^{2+}$-triggered vesicle fusion. Nat. Struct. Mol. Biol. 17, 325-331 (2010).

19. Choi, U.B. et al. Single-molecule FRET-derived model of the synaptotagmin 1SNARE fusion complex. Nat. Struct. Mol. Biol. 17, 318-324 (2010).

20. Schaub, J.R., Lu, X., Doneske, B., Shin, Y.K. \& McNew, J.A. Hemifusion arrest by complexin is relieved by $\mathrm{Ca}^{2+}$-synaptotagmin I. Nat. Struct. Mol. Biol. 13, 748-750 (2006).

21. Xue, M., Ma, C., Craig, T.K., Rosenmund, C. \& Rizo, J. The Janus-faced nature of the C(2)B domain is fundamental for synaptotagmin-1 function. Nat. Struct. Mol. Biol. 15, 1160-1168 (2008).

22. Tang, J. et al. A complexin/synaptotagmin 1 switch controls fast synaptic vesicle exocytosis. Cell 126, 1175-1187 (2006).

23. Weber, T. et al. SNAREpins: minimal machinery for membrane fusion. Cell 92, 759-772 (1998)

24. van den Bogaart, G. et al. One SNARE complex is sufficient for membrane fusion. Nat. Struct. Mol. Biol. 17, 358-364 (2010)

25. Stein, A., Radhakrishnan, A., Riedel, D., Fasshauer, D. \& Jahn, R. Synaptotagmin activates membrane fusion through a $\mathrm{Ca}^{2+}$-dependent trans interaction with phospholipids. Nat. Struct. Mol. Biol. 14, 904-911 (2007).

26. Chicka, M.C., Hui, E., Liu, H. \& Chapman, E.R. Synaptotagmin arrests the SNARE complex before triggering fast, efficient membrane fusion in response to $\mathrm{Ca}^{2+}$. Nat. Struct. Mol. Biol. 15, 827-835 (2008).

27. Lee, H.K. et al. Dynamic $\mathrm{Ca}^{2+}$-dependent stimulation of vesicle fusion by membraneanchored synaptotagmin 1. Science 328, 760-763 (2010).
28. Lynch, K.L. et al. Synaptotagmin C2A loop 2 mediates $\mathrm{Ca}^{2+}{ }^{-}$-dependent SNARE interactions essential for $\mathrm{Ca} 2+-$ triggered vesicle exocytosis. Mol. Biol. Cell 18, 4957-4968 (2007)

29. Tucker, W.C., Weber, T. \& Chapman, E.R. Reconstitution of $\mathrm{Ca}^{2+}$-regulated membrane fusion by synaptotagmin and SNAREs. Science 304, 435-438 (2004).

30. Rhee, J.S. et al. Augmenting neurotransmitter release by enhancing the apparent $\mathrm{Ca}^{2}$ affinity of synaptotagmin 1. Proc. Natl. Acad. Sci. USA 102, 18664-18669 (2005).

31. Margittai, M., Fasshauer, D., Pabst, S., Jahn, R. \& Langen, R. Homo- and heterooligomeric SNARE complexes studied by site-directed spin labeling. J. Biol. Chem. 276, 13169-13177 (2001).

32. Fasshauer, D. \& Margittai, M. A transient N-terminal interaction of SNAP-25 and syntaxin nucleates SNARE assembly. J. Biol. Chem. 279, 7613-7621 (2004).

33. Parlati, F. et al. Rapid and efficient fusion of phospholipid vesicles by the alphahelical core of a SNARE complex in the absence of an N-terminal regulatory domain. Proc. Natl. Acad. Sci. USA 96, 12565-12570 (1999).

34. Pobbati, A.V., Stein, A. \& Fasshauer, D. N- to C-terminal SNARE complex assembly promotes rapid membrane fusion. Science 313, 673-676 (2006).

35. Siddiqui, T.J. et al. Determinants of synaptobrevin regulation in membranes Mol. Biol. Cell 18, 2037-2046 (2007).

36. Li, F. et al. Energetics and dynamics of SNAREpin folding across lipid bilayers. Nat. Struct. Mol. Biol. 14, 890-896 (2007).

37. Holt, M., Riedel, D., Stein, A., Schuette, C. \& Jahn, R. Synaptic vesicles are constitutively active fusion machines that function independently of $\mathrm{Ca}^{2+}$ Curr. Biol. 18, 715-722 (2008).

38. James, D.J., Khodthong, C., Kowalchyk, J.A. \& Martin, T.F. Phosphatidylinositol 4,5-bisphosphate regulates SNARE-dependent membrane fusion. J. Cell Biol. 182 355-366 (2008)

39. van den Bogaart, G., Hermans, N., Krasnikov, V., de Vries, A.H. \& Poolman, B. On the decrease in lateral mobility of phospholipids by sugars. Biophys. J. 92, 1598-1605 (2007)

40. Cerjan, C. \& Barnett, R.E. The viscosity dependence of a putative diffusion-limited reaction. J. Phys. Chem. 76, 1192-1195 (1971).

41. Knight, J.B., Vishwanath, A., Brody, J.P. \& Austin, R.H. Hydrodynamic focusing on a silicon chip: mixing nanoliters in microseconds. Phys. Rev. Lett. 80, 3863-3866 (1998).

42. Takamori, S. et al. Molecular anatomy of a trafficking organelle. Cell 127, 831-846 (2006).

43. Nishiki, T. \& Augustine, G.J. Dual roles of the C2B domain of synaptotagmin I in synchronizing $\mathrm{Ca}^{2+}$-dependent neurotransmitter release. J. Neurosci. 24, 8542-8550 (2004).

44. Wienken, C.J., Baaske, P., Rothbauer, U., Braun, D. \& Duhr, S. Protein-binding assays in biological liquids using microscale thermophoresis. Nat. Commun. 1, 100 (2010).

45. Duhr, S. \& Braun, D. Why do molecules move along a temperature gradient? Proc. Natl. Acad. Sci. USA 103, 19678-19682 (2006).

46. Yoon, T.Y., Okumus, B., Zhang, F., Shin, Y.K. \& Ha, T. Multiple intermediates in SNARE-induced membrane fusion. Proc. Natl. Acad. Sci. USA 103, 19731-19736 (2006).

47. Borden, C.R., Stevens, C.F., Sullivan, J.M. \& Zhu, Y. Synaptotagmin mutants Y311N and K326/327A alter the calcium dependence of neurotransmission. Mol. Cell. Neurosci. 29, 462-470 (2005).

48. Marrink, S.J., Risselada, H.J., Yefimov, S., Tieleman, D.P. \& de Vries, A.H. The MARTINI force field: coarse grained model for biomolecular simulations. J. Phys. Chem. B 111, 7812-7824 (2007).

49. Monticelli, L. et al. The MARTINI coarse grained forcefield: extension to proteins J. Chem. Theory Comput. 4, 819-834 (2008).

50. Sutton, R.B., Ernst, J.A. \& Brunger, A.T. Crystal structure of the cytosolic C2A-C2B domains of synaptotagmin III. Implications for $\mathrm{Ca}^{2+}$-independent snare complex interaction. J. Cell Biol. 147, 589-598 (1999).

51. Hu, K. et al. Vesicular restriction of synaptobrevin suggests a role for calcium in membrane fusion. Nature 415, 646-650 (2002).

52. de Wit, H. et al. Synaptotagmin-1 docks secretory vesicles to syntaxin-1/SNAP-25 acceptor complexes. Cell 138, 935-946 (2009).

53. Walter, A.M., Wiederhold, K., Bruns, D., Fasshauer, D. \& Sørensen, J.B. Synaptobrevin N-terminally bound to syntaxin-SNAP-25 defines the primed vesicle state in regulated exocytosis. J. Cell Biol. 3, 401-413 (2010).

54. Borisovska, M. et al. v-SNAREs control exocytosis of vesicles from priming to fusion. EMBO J. 24, 2114-2126 (2005).

55. Gerber, S.H. et al. Conformational switch of syntaxin-1 controls synaptic vesicle fusion. Science 321, 1507-1510 (2008).

56. Schiavo, G., Stenbeck, G., Rothman, J.E. \& Söllner, T.H. Binding of the synaptic vesicle $v$-SNARE, synaptotagmin, to the plasma membrane t-SNARE, SNAP-25, can explain docked vesicles at neurotoxin-treated synapses. Proc. Natl. Acad. Sci. USA 3, 997-1001 (1997).

57. Mohrmann, R., de Wit, H., Verhage, M., Neher, E. \& Sørensen, J.B. Fast vesicle fusion in living cells requires at least three SNARE complexes. Science 6003 , 502-505 (2010)

58. Ernst, J.A. \& Brunger, A.T. High resolution structure, stability, and synaptotagmin binding of a truncated neuronal SNARE complex. J. Biol. Chem. 278, 8630-8636 (2003). 


\section{ONLINE METHODS}

Proteins were purified as described ${ }^{5,24,25}$. Liposomes were prepared by sizeexclusion chromatography as described ${ }^{24}$. Lipid mixing was performed as described $^{24}$, except that 1.5 mol\% 1,1'-dioctadecyl-3,3,3',3'-tetramethylindodicarbocyanine perchlorate (DiD; Invitrogen; emission $670 \mathrm{~nm}$ ) was used as an acceptor fluorophore to reduce cross-talk ${ }^{27}$. The lipid composition of the R-SNARE liposomes was chosen to mimic the synaptic vesicle ${ }^{42}$ and (unless stated otherwise) consisted of a 5:2:1:1 ratio of phospatidylcholine, phosphatidylethanolamine, phosphoserine and cholesterol (all lipids from Avanti Polar Lipids). These liposomes were labeled with 1.5 mol\% OG-PE (Invitrogen). The lipid composition of the DiD-labeled Q-SNARE liposomes was identical, except that $1 \mathrm{~mol} \%$ phospatidylcholine was replaced with the plasma membrane lipid PtdIns(4,5)P2. Unless stated otherwise, membrane fusion was triggered with $1 \mathrm{mM}$ $\mathrm{Ca}^{2+} \cdot \mathrm{Ca}^{2+}$ concentrations were calibrated with Fluo-5N and Mag-Fura-2 (Invitrogen). If not stated otherwise, all experiments at high ionic strength were performed in $20 \mathrm{mM}$ potassium HEPES, pH 7.4, $150 \mathrm{mM} \mathrm{KCl}$. For low ionic strength, the same buffer was used, but now with $5 \mathrm{mM} \mathrm{KCl}$ and $300 \mathrm{mM}$ sucrose. In all cases, lipid mixing could be well inhibited by competitive inhibition with either $10 \mu \mathrm{M}$ synaptobrevin- $2_{1-96}$ or with a combination of $10 \mu \mathrm{M}$ SNAP-25 and $10 \mu \mathrm{M}$ syntaxin-1 $\mathrm{A}_{183-263}$ (ref. 24).

Protocols have been described for the purification of synaptic vesicles from rat brain $^{37}$, calcein dequenching ${ }^{24}$, measurement of complex formation by FRET ${ }^{35}$ and fluorescence anisotropy ${ }^{34}$. In all cases, protein labeling efficiencies were $>80 \%$ as assessed by UV-visible spectroscopy ${ }^{24}$, except for syntaxin-1 $\mathrm{A}_{183-262} \mathrm{~S} 225 \mathrm{C}$, for which the labeling efficiency was only $\sim 30 \%$. EM was performed as described ${ }^{24}$, except that an additional wash step with $0.1 \%(\mathrm{w} / \mathrm{v})$ glutharaldehyde in water was performed to remove the sucrose. For the flow cytometry, microfluidic devices were produced using standard polydimethylsiloxane (PDMS) soft lithography ${ }^{41,59}$. Flow was regulated with home-built, computer-controlled pumps.
The emission at 540 and $610 \mathrm{~nm}$ was recorded simultaneously on a two-camera inverted fluorescence microscope setup. GUVs were prepared by the rehydration of lyophilized small liposomes ${ }^{60}$. Microscale capillary thermophoresis was measured with a NanoTemper NT.015, and $4 \mathrm{nM}$ of liposomes containing $5 \mathrm{~mol} \%$ OG-PE and $40 \mathrm{nM}$ of DiD-labeled liposomes. DLS was measured on a DynaPro (Wyatt Technology) with a total liposome concentration of $4 \mathrm{nM}$ in the case of full-length synaptotagmin-1 or $2 \mathrm{nM}$ liposomes in combination with $10 \mathrm{nM}$ of the soluble $\mathrm{C} 2 \mathrm{AB}$ fragment. For the FRET docking experiments ${ }^{46}$ (Fig. 4f,g), the DiI (1,1'-dioctadecyl-3,3,3',3'-tetramethylindocarbocyanine perchlorate; Invitrogen; $1.5 \mathrm{~mol} \%$; donor fluorophore) population of liposomes contained 1:4,000 synaptotagmin- 1 and the $\mathrm{DiD}$ ( $1.5 \mathrm{~mol} \%$; acceptor fluorophore) liposome population contained $1 \% \operatorname{PtdIns}(4,5) \mathrm{P} 2$.

For the molecular dynamics simulations, the MARTINI coarse-grained forcefield ${ }^{48,49}$ was applied to model synaptotagmin- 1 on the basis of the crystal structure $^{50}$. The simulation box of $7.3 \times 6.2 \times 15.0 \mathrm{~nm}$ contained a single $\mathrm{C} 2 \mathrm{AB}$ protein and 5,376 solvent particles. We performed simulations up to $3 \mu \mathrm{s}$ at $310 \mathrm{~K}$ while pulling pairwise with a constant velocity of $10^{-5} \mathrm{~nm} \mathrm{ps}^{-1}$ on the $\mathrm{N}$ terminus (residue 157), the polybasic lysine patch (234-237) and the two $\mathrm{Ca}^{2+}$-binding sites of the C2A $(172,178,230,232$ and 238) and C2B domains $(303,309,363$, 365 and 371). We fixed the structure of the $\mathrm{C} 2 \mathrm{~A}$ and $\mathrm{C} 2 \mathrm{~B}$ domains with an elastic network that connected all residues within $0.5-0.9 \mathrm{~nm}$ in the starting structure by a harmonic bond with a force constant of $500 \mathrm{~kJ} \mathrm{~mol}^{-1} \mathrm{~nm}^{-2}$. The center of mass of the binding sites was used to calculate the distances. Distances were calculated shortly before overstretching of the linker, which was apparent from a strong and continuous increase in the bond energy.

59. Cooper McDonald, J. et al. Fabrication of microfluidic systems in poly(dimethylsiloxane). Electrophoresis 21, 27-40 (2000).

60. Doeven, M.K. et al. Distribution, lateral mobility and function of membrane proteins incorporated into giant unilamellar vesicles. Biophys. J. 88, 1134-1142 (2005). 